\title{
Reinforcement expectancy and trial spacing effects in delayed matching-to-sample by pigeons
}

\author{
ANGELO SANTI \\ Wilfrid Laurier University, Waterloo, Ontario, Canada \\ and \\ WILLIAM A. ROBERTS \\ The University of Western Ontario, London, Ontario, Canada
}

\begin{abstract}
Four experiments assessed the role of reinforcement expectancies in the trial spacing effect obtained in delayed matching-to-sample by pigeons. In Experiment 1, a differential outcome (DO) group received reinforcement with a probability of 1.0 for correct comparison responses following one sample stimulus and a probability of 0.2 for correct comparison responses following the other sample stimulus. The nondifferential outcome (NDO) group received reinforcement with a probability of 0.6 for correct responses to either stimulus. While matching accuracy was higher for the DO group than for the NDO group, both groups showed an equivalent decline in accuracy as the intertrial interval (ITI) duration was decreased. However, within the DO group, ITI duration affected performance on low-probability-of-reinforcement trials but not on high-probabilityof-reinforcement trials. In Experiment 2, delay interval (DI) duration was 5, 10, or $15 \mathrm{sec}$ and accuracy was higher for the DO group than for the NDO group at all DI durations. In addition, accuracy decreased similarly on high- and low-probability-of-reinforcement trials for the DO group as DI was increased. In Experiment 3, all birds were studied under DO conditions and ITI duration was manipulated along with DI duration. At the short DI duration, decreasing ITI duration had a detrimental effect on low-probability-of-reinforcement trials but no effect on high-probabilityof-reinforcement trials. At the long DI duration, decreasing ITI duration had detrimental effects on both types of trials. In Experiment 4, unsignaled ITI reinforcers disrupted accuracy when the DI was long and when the ITI was short. The applicability of scalar expectancy theory to these data is discussed.
\end{abstract}

Numerous studies have shown that choice delayed matching-to-sample (DMTS) performance by pigeons is degraded by decreases in the temporal separation of trials (Grant, 1975; Hogan, Edwards, \& Zentall, 1981; Maki, Moe, \& Bierley, 1977). The poorer performance obtained with short intertrial intervals (ITI) was initially viewed as being due to competing memories from previous trials (Grant, 1975; Maki et al., 1977). However, this explanation has been called into question by several recent findings. Roberts (1980) found that short ITIs disrupted performance on homogeneous DMTS trials as well as on standard DMTS trials. Homogeneous DMTS trials present the same sample stimulus on each trial, eliminating the

This research was supported by Grant A6378 from the Natural Sciences and Engineering Research Council of Canada to Angelo Santi and by Grant A7894 from the Natural Sciences and Engineering Research Council of Canada to William A. Roberts. The authors would like to thank Julie Savich, Colleen Hanemaayer, and Bill Reason for their assistance. Reprints may be obtained from Angelo Santi, Department of Psychology, Wilfrid Laurier University, Waterloo, Ontario, Canada N2L 3C5. possibility of competing sample memories. Although there is evidence that memories on trial $n-1$ do carry over the ITI and affect performance on trial $n$, both Roberts (1980) and Roitblat and Scopatz (1983) have shown that these are memories of the choice made on the previous trial rather than of the sample stimulus presented on the trial $\mathrm{n}-1$. More importantly, neither study found any evidence that the carryover of memories between trials interacts with ITI duration, thus weakening explanations of the trial spacing effect based on proactive inhibition (PI). Finally, Santi (1984) manipulated chamber illumination during the ITI and found that DMTS performance was disrupted by illumination at long ITIs but was unaffected by illumination at short ITIs. If the poorer DMTS performance at short ITIs was due to PI, then, on the basis of the results of delay interval illumination studies (Cook, 1980; Grant \& Roberts, 1976; Maki et al., 1977; Roberts \& Grant 1978; Thompson, Van Hemel, Winston, \& Pappas, 1983; Tranberg \& Rilling, 1980), one would expect that illumination per se or illumination change at short ITIs would facilitate the forgetting of stimuli from previous trials and 
produce a release from PI. The absence of such an effect in the study by Santi (1984) supports the conclusion that trial spacing effects are not due to PI.

Roberts and Kraemer $(1982,1984)$ have suggested that scalar expectancy theory, as developed by Gibbon (1977) and Gibbon and Balsam (1981), may be extended to account for the effects of temporal variables on DMTS performance. The theory was developed to account for the effect of temporal variables on the acquisition of autoshaping. It assumes that successive reinforcements establish a background level of reinforcement expectancy which is spread evenly over the average interval between reinforcements. Decreasing ITI duration increases the level of reinforcement expectancy conditioned to the background. The presentation of trial stimuli ending with reinforcement results in an expectancy being conditioned to the trial stimuli as well. Roberts and Kraemer (1982, 1984) treated delay interval (DI) duration as analogous to trial duration. Hence, increases in DI duration decrease the level of reinforcement expectancy conditioned to trial stimuli. Reinforcement expectancies are conditioned to the background and to the trial stimuli in a noncompetitive fashion. Performance but not conditioning depends on a ratio comparator mechanism which compares the level of expectancy during trial stimuli with the background level of expectancy. When the ratio of overall expectancy during a trial to overall background expectancy rises above threshold, keypecking occurs in an autoshaping procedure and pecking the correct comparison stimulus may somehow be facilitated in a DMTS procedure. For example, it might be argued that stronger trial expectancies of reward relative to background expectancies promote better attention to sample stimuli and/or greater rehearsal of memorial representations.

Empirically, the effects of temporal variables on autoshaping acquisition are parallel to those on DMTS performance. DMTS accuracy, like autoshaping acquisition, increases as a direct linear function of the log ITI/delay ratio (Roberts \& Kraemer, 1982; Santi, 1984; Wilkie, 1984). It is also controlled more by the average ITI than by localized ITI values (Roberts \& Kraemer, 1982). In addition, free reinforcers presented during the ITI disrupt autopecking (Durlach, 1983; Jenkins, Barnes, \& Barrera, 1981) and also decrease DMTS accuracy (Spetch, 1984; Wilkie, 1984). The parallel empirical effects are provocative because they suggest that similar mechanisms may be operative in autoshaping and DMTS performance. Reinforcement expectancy is the mechanism by which scalar expectancy theory accounts for the effects of temporal variables on autoshaping. However, the development of a quantitative account of trial spacing effects in DMTS from a scalar expectancy theory perspective may be premature, since some of the empirical findings are not consistent with the theory. In a study of delayed matching of key location by pigeons, Wilkie (1984) showed that signaling ITI reinforcement by the correct comparison, the incorrect comparison, or an irrelevant stimulus for the upcoming trial facilitated performance, whereas un- signaled ITI reinforcement disrupted performance. According to scalar expectancy, signaling ITI reinforcers should not affect the conditioning of expectation to background stimuli (Gibbon \& Balsam, 1981; Jenkins et al., 1981). Hence, similar levels of matching accuracy should have been observed whether ITI reinforcement was signaled or not. Wilkie's (1984) result suggests that the background and trial stimuli compete for reinforcement expectancy or associative strength and that, therefore, signaling ITI reinforcement blocks the development of expectancy to the background. Spetch (1984) has shown that unsignaled reinforcers presented later in the ITI disrupt performance more than when presented early and that one reinforcer presented late in the ITI is as disruptive as the presentation of three reinforcers during the ITI. This sensitivity to the temporal location of ITI reinforcers as well as an insensitivity to the frequency of ITI reinforcers is difficult to reconcile with a scalar expectancy theory account of trial spacing effects.

Thus, the empirical findings to date are somewhat equivocal with respect to the appropriateness of extending scalar expectancy theory to DMTS performance. Nevertheless, thinking about DMTS performance in the context of scalar expectancy theory can serve a heuristic function. For example, it suggests that directly manipulating reinforcement expectancies during trial stimuli might alter the sensitivity of performance on these trials to spacing effects. One way of altering trial reinforcement expectancies is through a differential outcome procedure. A number of studies have demonstrated enhanced acquisition of discrimination and superior memory performance in pigeons when sample stimuli were associated with different trial outcomes (DO) following correct responding relative to when the outcomes were nondifferential (NDO). The DO effect has been demonstrated with qualitatively different reinforcers (Brodigan \& Peterson, 1976; Edwards, Jagielo, Zentall, \& Hogan, 1982; Honig, Matheson, \& Dodd, 1984; Peterson, Wheeler, \& Armstrong, 1978), reward versus no reward (Peterson, 1984; Peterson \& Trapold, 1980; Peterson, Wheeler, \& Trapold, 1980), and differential probability of reward (DeLong \& Wasserman, 1981). Several explanations of the DO effect have been proposed and tested. The hypothesis with the greatest degree of support maintains that differential outcome expectancies generated by the sample stimuli act as mediating cues and control responding to the comparison stimuli (Edwards et al., 1982; Honig et al., 1984; Peterson, 1984). This is consistent with the notion that the contents of working memory are prospective representations of anticipated outcomes (Honig \& Thompson, 1982).

\section{EXPERIMENT 1}

In the first experiment reported here, two groups of subjects were studied: a DO group and a NDO group. Probability of reinforcement for correct responding was the differential outcome, and it was anticipated that this would 
result in differential reinforcement expectancies for the two trial types in the DO group and nondifferential reinforcement expectancies in the NDO group. Specifically, for the DO group the probabilities of reinforcement were 1.0 and 0.2 , whereas for the NDO group they were both 0.6 . Thus, the overall probability of reinforcement in the presence of trial stimuli would be equivalent in the DO and NDO groups. The DO effect reported by DeLong and Wasserman (1981) with differential probability of reward utilized a successive DMTS procedure. In the present experiment, a choice DMTS procedure was used in order to determine whether differential probability of reward would enhance performance with this procedure as well. Both the DO group and the NDO group were tested at ITI durations of 1,5 , and $25 \mathrm{sec}$ and DI durations of 5 and $7 \mathrm{sec}$. If the manipulation of trial reinforcement expectancies through a differential outcome procedure alters the sensitivity of performance to trial spacing effects, then, within the DO group, the effect of ITI duration might differ for high- and low-probability-of-reinforcement trials.

\section{Method}

Subjects. Eight White Carneaux pigeons, maintained at $80 \%$ $\pm 20 \mathrm{~g}$ of their ad-lib weight and housed individually with constant access to grit and water, served as subjects. The colony room was lighted from 6 a.m. to 7 p.m., and the subjects were run between the hours of 8 and 11 a.m., one session per day, 5 days per week. All of the subjects had had previous experience with various choice DMTS procedures.

Apparatus. Four Coulbourn modular operant test cages (Model E10-10) were housed individually in isolation cubicles (Model E10-20). Each cubicle was equipped with a ventilation fan and baffled air intake and exhaust system. Each test cage was equipped with three clear plastic pecking keys, behind which projectors could display stimuli (red, green, and a black dot on a white background) onto a frosted rear-projection screen (Coulbourn Model E21-18). The three pecking keys were horizontally aligned on the front panel. Directly below the center key was a $5.7 \times 5 \mathrm{~cm}$ opening which provided access to a hopper filled with mixed grain (Coulbourn Model E14-10). All experimental stimuli and response measures were arranged and recorded by a microcomputer system located in an adjacent room.

Procedure. Prior to testing, all birds received 18 sessions of training on a choice DMTS task involving highly familiar sample stimuli. Each trial began with a warning signal (a black dot on a white background) presented on the center key. A peck to it produced either a red or a green sample stimulus. The sample stimulus was terminated after $5 \mathrm{sec}$ and followed by a DI of $1.5 \mathrm{sec}$ in the first 10 sessions and one of $4 \mathrm{sec}$ in the last 8 sessions of baseline training. At the end of the DI, red and green comparison stimuli were presented on the side keys with positions counterbalanced over trials. A single peck to the comparison stimulus that matched the sample was defined as a correct response and resulted in termination of the comparison stimuli. For four birds (Group NDO), the probability of food reinforcement (3-sec access to mixed grain) was 0.6 following correct responses to either red or green stimuli. For the other four birds (Group DO), the probability of food reinforcement was 1.0 following correct responses to one stimulus and 0.2 following correct responses to the other stimulus. For two of the Group DO birds, the probability of reinforcement for correct responses was 1.0 for trials involving a red sample; for the other two birds, it was 1.0 for trials involving a green sample. For either Group NDO or Group DO, on trials when reinforcement did not occur, termination of the comparison stimuli was followed by a 3-sec blackout. Following either reinforcement or blackout, an ITI of $15 \mathrm{sec}$, spent in darkness, occurred. The only illumination provided in the chamber was produced by illumination of the key lights or the bulb in the grain feeder. Each session consisted of 100 trials ( 25 individually randomized blocks of the four different stimulus configurations).

Testing was carried out in two blocks of 6 days each. There were six treatment conditions defined by the factorial combination of ITI duration $(1,5$, or $25 \mathrm{sec})$ and DI duration $(5$ or $7 \mathrm{sec})$. Only one treatment condition was presented on a given test day. Within each block of 6 days, each of the six treatment conditions was tested once, with the sequence of testing randomized individually for each bird. Except for the changes introduced by the manipulated variables, all aspects of a test session were identical to those described for training sessions. The .05 level of significance was adopted for all of the statistical tests reported.

\section{Results}

Over the last 3 days of baseline training, Group DO averaged $96.9 \%$ correct and Group NDO averaged $88.4 \%$ correct. This superiority in performance for Group DO was statistically significant $[\mathrm{t}(6)=2.09$ with a one-tailed test]. Figure 1 summarizes matching accuracy during testing. The panel on the left is for test data obtained with a DI of $5 \mathrm{sec}$, and the one on the right is for data obtained with a DI of $7 \mathrm{sec}$. An analysis of variance of these data revealed that accuracy was higher in Group DO than in Group NDO $[\mathrm{F}(1,6)=6.05, \mathrm{MSe}=375.40]$ and that accuracy increased as a function of ITI duration $[F(2,12)=11.26, \mathrm{MSe}=77.71]$. No other effects were statistically significant.

The data for Group DO were analyzed further by an examination of performance on high- and low-probability-of-reinforcement trials. These data are presented in Figure 2. Accuracy on high-probability-of-reinforcement trials during testing is indicated by the filled circles and solid lines; accuracy on low-probability-of-reinforcement trials is indicated by unfilled circles and dashed lines. The data indicate that the trial spacing effect is much more evident for low-probability-of-reinforcement trials, especially at the lower DI value. An analysis of variance indicated a significant effect of ITI duration $[F(2,6)=7.43$, $\mathrm{MSe}=63.46]$ and a significant interaction of ITI duration

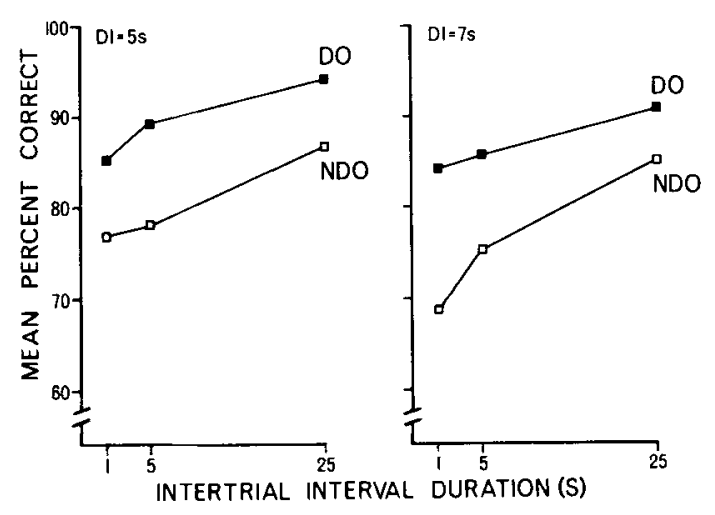

Figure 1. Mean percentage of correct responses during testing as a function of intertrial interval duration and delay interval (DI) for differential outcome (DO) and nondifferential outcome (NDO) groups in Experiment 1. 


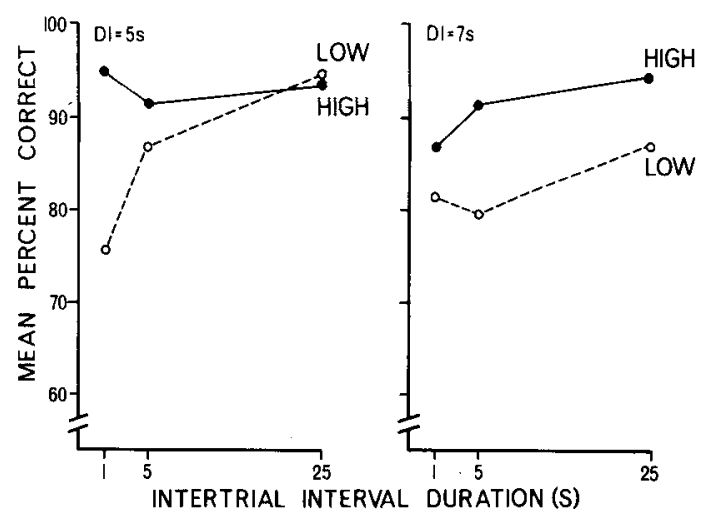

Figure 2. Mean percentage of correct responses during testing as a function of intertrial interval duration and delay interval (DI) for high- and low-probability-of-reinforcement trials in Experiment 1.

and reinforcement probability $[\mathrm{F}(2,6)=7.03$, MSe $=$ 23.82]. A simple main effect analysis of this interaction indicated a significant effect of ITI duration for lowprobability-of-reinforcement trials $[\mathrm{F}(2,6)=25.11]$ but not for high-probability-of-reinforcement trials $[F(2,6)=$ 1.68]. Furthermore, accuracy was higher on high-probability trials than on low-probability trials at the 1- and 5 -sec ITI durations $[\mathrm{F}(1,6)=50.40$ and 22.51$]$ but not at the 25-sec duration $[\mathrm{F}(1,6)=3.28]$.

An analysis of responses to the sample stimuli was undertaken. The mean number of sample responses per session for Group DO is presented in Table 1. The mean number of responses to the sample associated with the higher probability of reinforcement was significantly higher than the mean number of responses to the sample associated with the low probability of reinforcement $[\mathrm{F}(1,3)=10.12, \mathrm{MSe}=51985.98]$. In addition, the mean number of responses to the sample stimuli in a session increased as a function of ITI duration $[F(2,6)=$ 8.59, $\mathrm{MSe}=8046.70$ ] for both high- and low-probability-of-reinforcement trials. The interaction of ITI duration and reinforcement probability was not significant $[F(2,6)=3.16]$. An analysis of sample responding for Group NDO showed no significant effects of ITI. In general, conditions that produced high levels of matching accuracy also produced higher levels of sample responding. However, the relationship between sample responding and choice accuracy did not appear to be very strong.

\section{Discussion}

The present results add to the literature on differential outcomes and trial spacing effects in several ways. First,

Table 1

Mean Number of Sample

Responses Per Session in Experiment 1

\begin{tabular}{crrr}
\hline \multirow{2}{*}{$\begin{array}{c}\text { Probability of } \\
\text { Reinforcement }\end{array}$} & \multicolumn{3}{c}{ Intertrial Interval Duration } \\
\cline { 2 - 4 } & $1 \mathrm{sec}$ & $5 \mathrm{sec}$ & $25 \mathrm{sec}$ \\
\hline High & 210.4 & 240.9 & 267.6 \\
Low & 43.3 & 64.9 & 166.8 \\
\hline
\end{tabular}

they reveal that the DO effect obtained by DeLong and Wasserman (1981) with differential probability of reward in a successive DMTS procedure is generalizable to choice DMTS procedures. Second, the results reveal trial spacing effects in terms of overall accuracy for both Group DO and Group NDO. This indicates that trial spacing effects are not dependent on the availability of reinforcement on every trial, but can be demonstrated in a task with probabilistic, nondifferential reinforcement. Finally, and most importantly, the results indicate that the overall trial spacing effect in Group DO was due to the effects of trial spacing on low-probability-of-reinforcement trials. No significant effect of trial spacing on accuracy was evident on high-probability-of-reinforcement trials. However, trial spacing did affect sample responding in a similar fashion on both high- and low-probability-of-reinforcement trials in Group DO.

Since equivalent trial-spacing effects were obtained with respect to sample responding on both high- and low-probability trials in Group DO, it was possible that the accuracy measure was either insensitive to or biased against revealing a trial spacing effect for high-probability trials. Since accuracy reflects a choice between a frequently reinforced and an infrequently reinforced comparison stimulus in the current procedure, it might be anticipated that a response bias exists in favor of the high-probability-ofreinforcement stimulus. One argument against this notion was the finding that accuracy on high- and low-probability trials was equivalent at the longest ITI duration tested. However, it may be that any response bias induced by this procedure is evidenced only when the task is made somewhat difficult. The next experiment was undertaken to evaluate this possibility empirically.

\section{EXPERIMENT 2}

In this experiment, birds in both Group DO and Group NDO were tested at relatively long DIs $(5,10$, or $15 \mathrm{sec})$. If a response bias was responsible for the lack of an effect of ITI duration on high-probability trials in the previous study, then one might anticipate either a lack of an effect of DI or a shallower forgetting curve for highprobability trials than for low-probability trials in the present experiment. That is, if the experimental procedure encourages birds to select the comparison stimulus associated with the higher probability of reinforcement, then, as the DI is increased and information derived from the sample is less accessible, there should be an increased tendency to select the high-probability-of-reinforcement stimulus. A significant interaction of trial type and DI duration should be obtained. The absence of such an interaction would suggest that the lack of a trial spacing effect for high-probability trials in the previous study was not due to response bias.

\section{Method}

Subjects and Apparatus. The subjects and apparatus were the same as for Experiment 1. 
Procedure. Following the testing described in Experiment 1, the birds were returned to the baseline conditions that preceded that experiment. The ITI was $15 \mathrm{sec}$, the sample duration was $5 \mathrm{sec}$, and the DI was $4 \mathrm{sec}$. All aspects of a trial were exactly as described for the baseline training sessions of Experiment 1 . All birds were given 11 sessions of baseline training. Testing was carried out in five blocks of 3 days each. Only one of the three DI durations studied $(5,10$, or $15 \mathrm{sec})$ was tested on a given day. Within each block of 3 days, each of the three DI durations was tested once, with the sequence of testing randomized individually for each bird.

\section{Results}

Over the last 3 days of baseline training, Group DO averaged $95.25 \%$ correct and Group NDO averaged $90 \%$. This difference was not statistically significant $[\mathrm{t}(6)=$ 1.67]. Overall accuracy during testing is presented in the upper panel of Figure 3. Performance in both groups decreased significantly as a function of DI duration $[\mathrm{F}(2,12)=31.75, \mathrm{MSe}=87.67]$; however, the performance of the DO group was superior to that of the NDO group at all durations tested $[\mathrm{F}(1,6)=8.56$, MSe $=$ 577.26]. The interaction of outcome group and DI was not significant $[F(2,12)=1.08]$. The analysis of variance also revealed a significant effect of test block $[\mathrm{F}(4,24)=3.00, \mathrm{MSe}=56.89]$ and a significant interaction of test block and delay $[\mathrm{F}(8.48)=2.51, \mathrm{MSe}=$ 36.47]. This interaction reflected a significant improve-
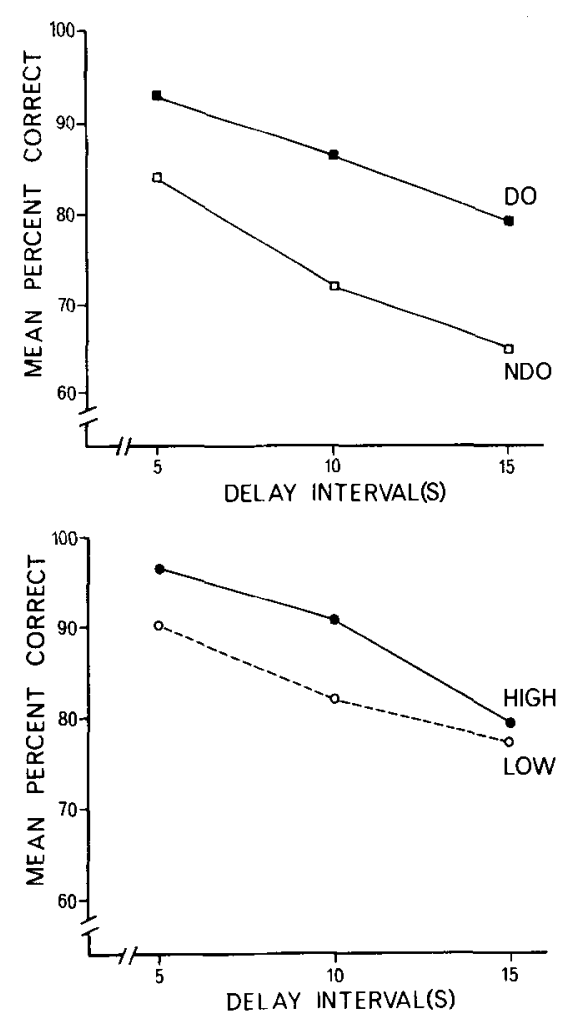

Figure 3. Mean percentage of correct responses as a function of delay interval in Experiment 2. The upper panel presents the data for the differential outcome (DO) and nondifferential outcome (NDO) groups; the lower panel presents the data obtained from the highand low-probability-of-reinforcement trials in the DO group.
Table 2

Mean Number of Sample Responses Per Session in Experiment 2

\begin{tabular}{crrr}
\hline Probability of & \multicolumn{3}{c}{ Delay Interval Duration } \\
\cline { 3 - 4 } Reinforcement & $5 \mathrm{sec}$ & $10 \mathrm{sec}$ & $15 \mathrm{sec}$ \\
\hline High & 192.4 & 230.2 & 243.8 \\
Low & 77.9 & 87.0 & 82.6 \\
\hline
\end{tabular}

ment in performance at the 10- and 15-sec DIs over test blocks, whereas performance at the 5-sec DI was higher and stable over test blocks. None of the remaining interaction terms was significant in the overall analysis.

Performance on high- and low-probability-of-reinforcement trials is presented for the DO group in the lower panel of Figure 3. Performance on high-probability trials was superior to that on low-probability trials $[F(1,3)=$ $11.09, \mathrm{MSe}=84.80]$, and performance on both trial types decreased as DI duration increased $[\mathrm{F}(2,6)=7.66$, $\mathrm{MSe}=288.50 \mathrm{]}$. Most importantly, there was no indication of a significant DI and reinforcement probability interaction $[\mathrm{F}(2,6)=1.66]$. The loss of information as a function of DIs was not accompanied by a response bias in favor of the most frequently reinforced comparison. There was a significant interaction of reinforcement probability and test blocks, which occurred as a result of equivalent levels of accuracy on high- and low-probability trials on the first test block, followed by a significant increase in performance on the high-probability trials in the remaining test blocks; performance on low-probabilityof-reinforcement trials did not change $[\mathrm{F}(4,12)=5.49$, MSe $=3.16$ ].

An analysis of the total number of sample responses emitted in a session was undertaken for Group DO. These data are presented in Table 2. More pecks were made to the sample stimulus associated with the high probability of reinforcement $[F(1,3)=17.82$, MSe $=32837.23]$. The number of pecks to the sample stimulus increased as a function of $\mathrm{DI}[\mathrm{F}(2,6)=7.92, \mathrm{MSe}=1140.90]$. This was more evident for high-probability than for low-probability trials, but the interaction of DI and reinforcement probability was not statistically significant $[F(2,6)=$ 2.82]. The number of sample responses decreased significantly over test blocks $[\mathrm{F}(4,12)=4.13$, MSe $=$ 1362.64].

\section{Discussion}

The present results indicate that a response bias was not responsible for the lack of an ITI duration effect on high-probability-of-reinforcement trials in Experiment 1. If a response bias had been encouraged by the present experimental procedures, then one would have anticipated increased selection of the most frequently reinforced comparison stimulus as DI duration was increased. This did not occur. Performance declined similarly on high- and low-probability-of-reinforcement trials as a function of DI duration.

As was the case in Experiment 1, Experiment 2 showed that the findings of DeLong and Wasserman (1981) could 
be replicated with a choice procedure. The findings of Experiment 1 were extended in Experiment 2 by showing that superior performance with differential outcomes occurred at all retention intervals. Furthermore, the results indicate that both the high- and low-reinforcement trials contributed to the overall higher accuracy for Group DO relative to Group NDO. Accuracy on low-probability trials was 90.2, 82.0, and 77.4 at the three DIs tested; for Group NDO, accuracy was $84.0,71.9$, and 64.6 at the three DIs. This difference in favor of the lower probability of reinforcement in the differential outcome condition was also reported by DeLong and Wasserman. However, unlike their study, the present results revealed clearly superior performance on high-relative to lowprobability-of-reinforcement trials. Both studies found higher rates of sample responding on high-probability-ofreinforcement trials. Thus, the difference in results in the accuracy measure may reflect differences in the nature of the dependent variable in the two studies. In the present choice DMTS procedure, accuracy reflects a choice between the high- and low-probability stimuli, whereas in the successive DMTS procedure employed by DeLong and Wasserman accuracy reflects response rate to a single test stimulus. It may be that a choice DMTS procedure is more sensitive to reinforcement probability differences than is a successive DMTS procedure.

\section{EXPERIMENT 3}

This experiment was conducted to provide more convincing data with respect to the replicability of the results of Experiment 1. The four birds studied under the NDO condition in the two previous studies were shifted to the DO condition. This resulted in eight birds tested under the DO condition in Experiment 3. During testing, both ITI duration (1, 5, or $25 \mathrm{sec}$ ) and DI duration (5 or $15 \mathrm{sec}$ ) were manipulated. A statistically more powerful analysis of the effects of ITI duration and DI duration on highand low-probability-of-reinforcement trials could be undertaken. On the basis of previous findings, it was expected that performance would be greater on high-probability trials than on low-probability trials. At the shorter DI, an effect of ITI duration was expected for low-probability trials but not for high-probability trials. The longer DI duration was incorporated into the present experiment to determine whether a trial spacing effect might be obtained for high-probability trials at a longer DI than had previously been used.

\section{Method}

Subjects and Apparatus. The subjects and apparatus were the same as for Experiment 2.

Procedure. Following the testing in Experiment 2, the birds were returned to the baseline conditions for four sessions. Then four birds in Group NDO were shifted to a differential outcome procedure identical to that previously described for Group DO. All eight birds continued to be run on the differential outcome procedure for a total of 13 sessions. All aspects of a trial were exactly as described for Group DO prior to Experiment 2. Testing was carried out in two blocks of 6 days each. There were six treatment conditions defined by the factorial combination of ITI duration $(1,5$, or $25 \mathrm{sec})$ and
DI duration $(5$ or $15 \mathrm{sec})$. All aspects of testing were similar to those described for Experiment 1.

\section{Results}

The data obtained when the four NDO birds were shifted to a DO procedure are shown in Figure 4. Relative to the NDO baseline, accuracy increased on the highprobability-of-reinforcement trials and initially decreased on the low-probability-of-reinforcement trials. Over sessions, performance on the low-probability-of-reinforcement trials improved. On the last session of baseline training, performance on low-probability-of-reinforcement trials was only slightly below that on high-probability-ofreinforcement trials. Over these baseline sessions, the original DO group maintained a very high and stable level of performance, comparable to that achieved by the shifted group on the last session of training. An analysis of sample responding for the group shifted from NDO to DO conditions revealed an overall increase in sample responding as a function of the shift, which was maintained over sessions. In addition, for three birds, there was a higher rate of sample responding to the high-probability-of-reinforcement stimulus. On the last session of training, six of eight birds emitted $63 \%$ or more of sample responses to the high-probability stimulus. The remaining two birds showed no clear preference.

Matching accuracy during testing is summarized in Figure 5. The panel on the left is for test data obtained with a DI of $5 \mathrm{sec}$; the one on the right is for data obtained with a DI of $15 \mathrm{sec}$. Separate analyses of variance were conducted for the data at each delay. At the DI of $5 \mathrm{sec}$, accuracy was greater on high-probability trials than on low-probability trials $[F(1,7)=14.65, \mathrm{MSe}=40.14]$ and accuracy increased as a function of ITI duration $[\mathrm{F}(2,14)=8.15, \mathrm{MSe}=105.63]$. In addition, there was

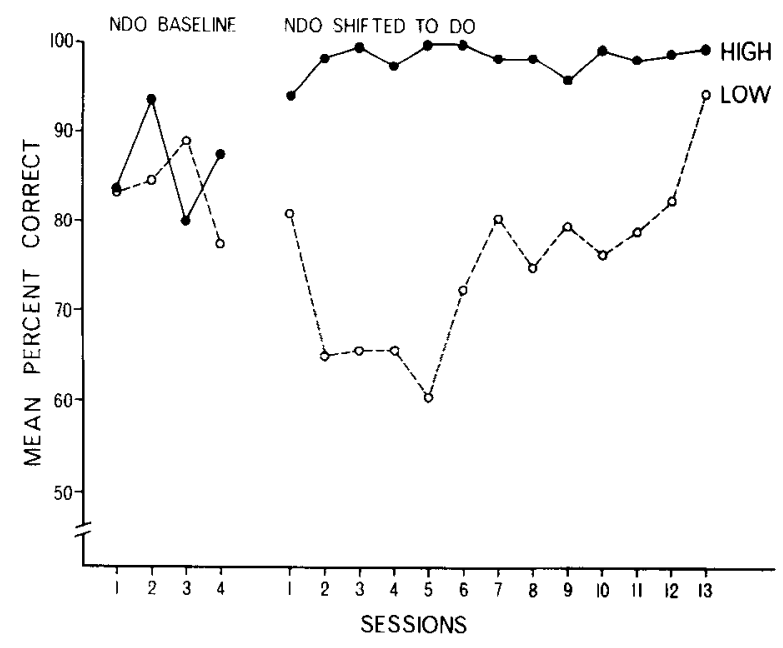

Figure 4. Mean percentage of correct responses for the nondifferential outcome (NDO) group prior to and following the shift to differential outcome (DO) conditions. The filled circles indicate performance on the high-probability-of-reinforcement trials following the shift and comparable control trials prior to the shift. The unfilled circles represent similar data for low-probability-ofreinforcement trials. 


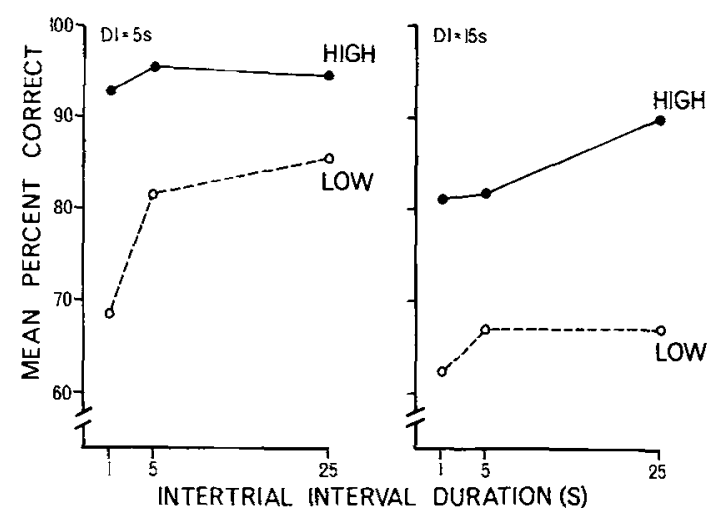

Figure 5. Mean percentage of correct responses during testing as a function of intertrial-interval duration and delay interval (DD) for high- and low-probability-of-reinforcement trials in Experiment 3.

a significant interaction of ITI duration and reinforcement probability $[\mathrm{F}(2,14)=4.09, \mathrm{MSe}=121.17]$. A simple main effect analysis of this interaction indicated a significant effect of ITI duration for low-probability trials $[\mathrm{F}(2,14)=11.19]$ but not for high-probability trials $(F<1)$. At the DI of $15 \mathrm{sec}$, accuracy was greater on high-probability trials than on low-probability trials $[\mathrm{F}(1,7)=13.09, \mathrm{MSe}=653.33]$, and accuracy increased as a function of ITI duration $[\mathrm{F}(2,14)=3.93$, $\mathrm{MSe}=$ 95.52]. Most importantly, there was no significant interaction of ITI duration and reinforcement probability $(\mathrm{F}<1)$. Hence, at the longer delay, trial spacing effects were obtained on both high- and low-probability-of-reinforcement trials.

The mean number of sample responses per session during testing is presented in Table 3 . At the DI of $5 \mathrm{sec}$, the only significant effect obtained resulted from the greater number of sample responses on high-probability-of-reinforcement trials $[\mathrm{F}(1,7)=6.47$, MSe $=$ 62734.45]. At the DI of $15 \mathrm{sec}$, both the effect of reinforcement probability $[F(1,7)=21.79$, MSe $=12016.66]$ and the interaction of ITI duration and reinforcement probability $[\mathrm{F}(2,14)$, $\mathrm{MSe}=2527.30]$ were significant . A simple main effect analysis revealed that ITI duration significantly affected sample responding only on lowprobability-of-reinforcement trials $[F(2,14)=5.55]$; no significant effect was obtained on high-probability-ofreinforcement trials $[F(2,14)=1.12]$. In addition, a significantly greater number of sample responses were observed on high-probability-of-reinforcement trials at each of the ITI durations tested.

Table 3

Mean Number of Sample

Responses Per Session in Experiment 3

\begin{tabular}{|c|c|c|c|c|}
\hline \multirow{2}{*}{$\begin{array}{c}\text { Delay } \\
\text { Interval }\end{array}$} & \multirow{2}{*}{$\begin{array}{l}\text { Probability of } \\
\text { Reinforcement }\end{array}$} & \multicolumn{3}{|c|}{ Intertrial Interval Duration } \\
\hline & & $1 \mathrm{sec}$ & $5 \sec$ & $25 \mathrm{sec}$ \\
\hline \multirow{2}{*}{$5 \mathrm{sec}$} & High & 238.0 & 249.0 & 200.0 \\
\hline & Low & 98.3 & 90.1 & 108.6 \\
\hline \multirow[b]{2}{*}{$15 \mathrm{sec}$} & High & 211.5 & 194.7 & 185.3 \\
\hline & Low & 76.0 & 75.2 & 126.9 \\
\hline
\end{tabular}

\section{Discussion}

As in Experiment 1, the manipulation of trial reinforcement expectancies through a differential outcome procedure altered the sensitivity of performance on those trials to ITI duration. At the shorter DI, a trial spacing effect was obtained for low-probability-of-reinforcement trials but not for high-probability-of-reinforcement trials. At the longer DI duration, a trial spacing effect was evident for both types of trial. At both DI durations, performance was better on high- than on low-probability-of-reinforcement trials and sample responding was higher as well. However, when one considers the way in which accuracy and sample responding were affected by probability of reinforcement, ITI, and DI duration jointly, the relationship between the two measures does not appear to be very strong in either Experiment 1 or Experiment 3.

\section{EXPERIMENT 4}

As noted in the introduction to Experiment 1, unsignaled reinforcers presented during the ITI decrease DMTS accuracy (Spetch, 1984; Wilkie, 1984). Experiment 4 was undertaken to replicate this effect and to determine whether the effect of unsignaled ITI reinforcers was different for high- and low-probability-of-reinforcement trials. The study was carried out in two replications. Four birds received unsignaled ITI reinforcers and four did not receive ITI reinforcers. Testing was then carried out with the four treatment combinations of ITI duration ( 5 and $25 \mathrm{sec}$ ) and DI duration ( 5 and $15 \mathrm{sec}$ ). Following testing, the birds were given several baseline sessions and then reversed with respect to the ITI reinforcer variable. Following 12 baseline sessions, the birds were retested at the four treatment combinations of ITI and DI. As a result, test data were available from all eight birds in both the ITI-reinforcer and the no-ITI-reinforcer conditions.

\section{Method}

Subjects and Apparatus. The subjects and apparatus were the same as those used previously.

Procedure. Following the testing described in Experiment 3, a study was conducted in which, for four birds, ITI reinforcers were introduced in an illuminated chamber and no ITI reinforcers occurred in a darkened chamber. Chamber illumination was alternated between sessions. The relationship between chamber illumination and ITI reinforcement was reversed for the remaining four birds. The data from this study will not be reported since chamber illumination had a large disruptive effect on performance and this variable was confounded with the ITI reinforcement variable. Following this unreported study, all birds were given five sessions of training in which trial events occurred in a darkened chamber. All aspects of these sessions were the same as those for Experiment 3, except that four birds received unsignaled ITI reinforcers in the middle of a 15-sec ITI but the other four birds did not. ITI reinforcers like end-of-trial reinforcers consisted of 3-sec access to mixed grain. The ITI reinforcers were programmed to occur with a probability of 0.4 . Thus, two out of every five ITIs were randomly selected and the reinforcer was presented in the middle of these two ITIs. Consequently, 40 ITI reinforcers were delivered in each session. A value of 0.4 was selected because, in conjunction with the DO procedure used, the overall number of reinforcers available per session would then be equal to that available if the probability of reinforcement for correct responses was equal to 1.0 on both trials. 
Each session consisted of 100 trials. Twelve test sessions were then administered in which a particular combination of DI (5 or $15 \mathrm{sec})$ and ITI ( 5 or $25 \mathrm{sec}$ ) was tested once on a given day. Following the 12 test sessions, the birds were given 4 sessions of training with conditions the same as those preceding testing. Then ITI reinforcement conditions were reversed. The four birds that had received ITI reinforcers previously now received no ITI reinforcers, and those that previously had not received ITI reinforcers now received them. Twelve sessions of training were administered following this reversal, and then testing was carried out again as previously described.

\section{Results}

The baseline data were examined for effects associated with the presence of ITI reinforcers. These data are presented in Figure 6. Significant effects due to the presence of unsignaled ITI reinforcers that were occasionally presented in the middle of a 15 -sec ITI were not found [t $(6)=$ $1.25,<1,<1$, respectively]. An analysis of variance of the accuracy data from test sessions revealed better performance on high- than on low-probability-of-reinforcement trials $[\mathrm{F}(1,7)=8.91, \mathrm{MSe}=130.68]$, better performance at the shorter delay $[\mathrm{F}(1,7)=27.53, \mathrm{MSe}=$ 97.77], and better performance at the longer $\operatorname{ITI}[F(1,7)=$ 26.24, $\mathrm{MSe}=32.11]$. The ITI reinforcement variable interacted significantly with $\mathrm{DI}[\mathrm{F}(1,7)=14.97$, MSe $=$ $4.10]$ and with ITI $[F(1,7)=8.09$, MSe $=28.31]$. These interactions are presented in the upper and lower panel of Figure 7. Unsignaled ITI reinforcers significantly lowered performance at the long delay $[F(1,7)=6.13]$, but not at the short delay $[F(1,7)=3.19]$. In addition, unsignaled ITI reinforcers significantly reduced performance at the short ITI duration $[F(1,7)=6.50]$, but not at the long ITI duration $[F(1,7)=2.14]$. The overall analysis of variance also revealed a marginally significant probability of reinforcement $\times$ DI $\times$ ITI interaction $[\mathrm{F}(1,7)=4.21, \mathrm{MSe}=47.67, \mathrm{p}<.08]$, which is shown in Figure 8. For high-probability-of-reinforcement trials, the effect of ITI was not significant at the short delay $[F(1,7)=1.95]$, but it was marginally significant at the longer delay $[F(1,7)=5.19, p<.06]$. For low-

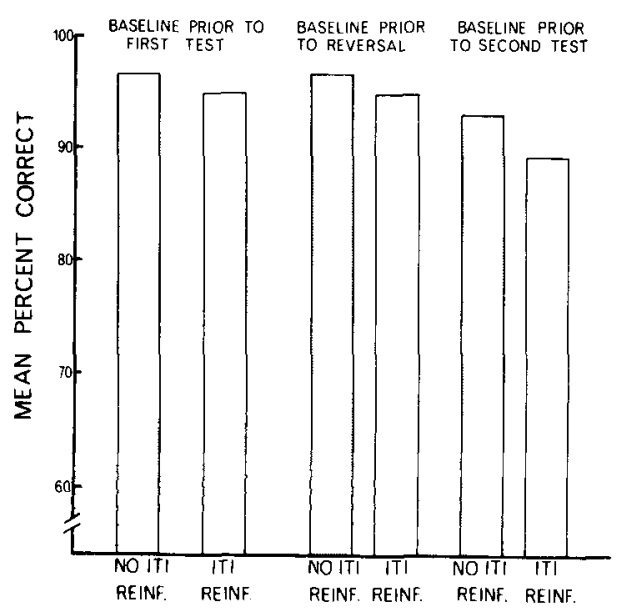

Figure 6. Mean percentage of correct responses for the baseline phases of Experiment 4.

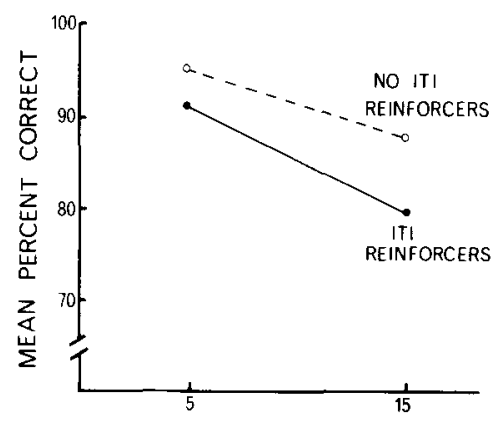

DELAY INTERVAL (sec)

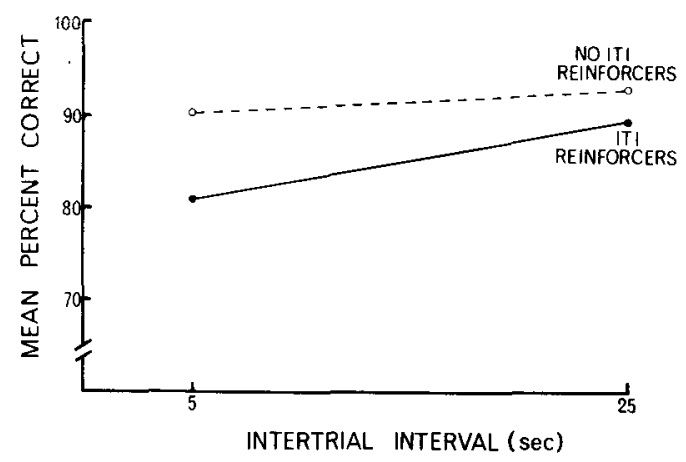

Figure 7. Mean percentage of correct responses during testing in Experiment 4. The upper panel presents the interaction of ITI reinforcement $x$ delay interval; the lower panel presents the interaction of ITI reinforcement $x$ intertrial interval duration.

probability-of-reinforcement trials, the effect of ITI was significant at the short delay $[F(1,7)=33.03]$ but not at the longer delay $[F(1,7)=3.25]$. The interaction of probability of reinforcement and the ITI reinforcement variable was not significant $[F<1]$.

\section{Discussion}

Despite marked procedural variations between the present study and Spetch's (1984) study, the effects of unsignaled ITI reinforcers were very similar. In both studies, disruptive effects of unsignaled ITI reinforcers occurred only at the longest DIs tested. The dependence of an effect of unsignaled ITI reinforcers on ITI duration in the present study is also consistent with her findings, which show that ITI reinforcers are disruptive only if they occur late in the ITI. At the short ITI, unsignaled reinforcers occurred in close temporal proximity to trials, whereas, at the long ITI, unsignaled ITI reinforcers were temporally more remote from trial events. Consequently, disruptive effects of ITI reinforcers were obtained only with the short ITI duration. This is also consistent with the failure to observe any effects of ITI reinforcers in the baseline sessions before and after testing. Since the ITI duration was $15 \mathrm{sec}$ and ITI reinforcers occurred in the middle of the ITI, they were temporally too remote from trial events to disrupt performance. Thus, temporal proximity to trial events is an important determiner of whether unsignaled ITI reinforcers will lower DMTS performance.

Although it was only marginally significant, the interaction of probability of reinforcement $\times$ DI $\times$ ITI re- 


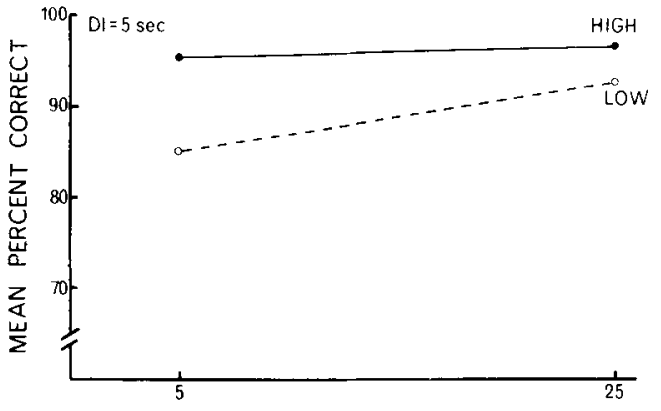

INTERTRIAL INTERVAL (sec)

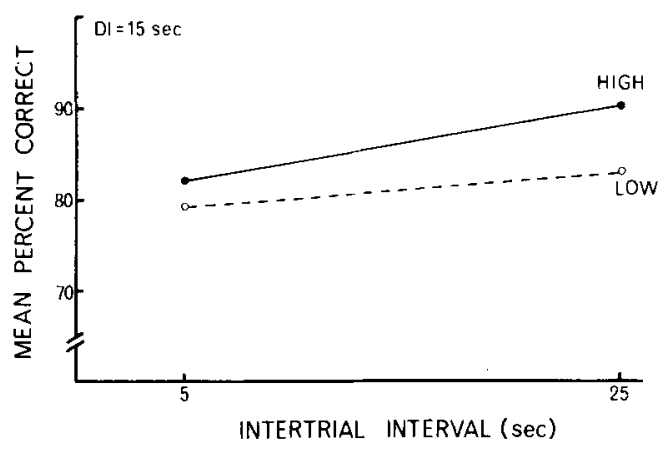

Figure 8. Mean percentage of correct responses during testing as a function of intertrial interval duration and delay interval (DI) for high- and low-probability-of-reinforcement trials in Experiment 4.

vealed a dependence of trial spacing effects on DI and reinforcement probability, as shown in Experiments 1 and 3. For high-probability-of-reinforcement trials, a trial spacing effect was obtained at the long delay but not at the short delay. For low-probability-of-reinforcement trials, a trial spacing effect was obtained at the short delay but not at the long delay. The failure to observe a trial spacing effect at the long delay for low-probability trials was puzzling. In part it may be due to the use of a 5-sec ITI rather than a 1-sec ITI as the shortest ITI duration. Because of the occurrence of ITI reinforcers, the shortest ITI duration used in the present experiment was $5 \mathrm{sec}$.

\section{GENERAL DISCUSSION}

Experiments 1 and 2 of the present study found superior matching accuracy by the group trained with differential reinforcement probabilities for correct responses. This constitutes an extended replication of results originally obtained by DeLong and Wasserman (1981) with a successive DMTS procedure, and is consistent with the notion that the contents of working memory are prospective representations of anticipated outcomes (Honig \& Thompson, 1982). In the present study, when differential reinforcement probabilities were employed as trial outcomes, both matching accuracy and the number of sample responses per session were higher on high-probabilityof-reinforcement trials than on low-probability-ofreinforcement trials. DeLong and Wasserman (1981) obtained a similar result with respect to sample responding, but found no difference in their accuracy measure, which was based on relative response rates to a single test stimulus. In their successive DMTS procedure, the occurrence of reinforcement was not dependent upon the pigeon's accuracy. In the present choice procedure, the occurrence of reinforcement depended on accurate responding. This methodological difference could have contributed to the differences in accuracy on high- and low-probability trials found in the present experiments. In addition, choice between the high- and low-probability stimuli may be a more sensitive index of reinforcement-expectancy differences than the discrimination ratio used in successive DMTS procedures. The results of Experiment 2 demonstrated that having to choose between a high- and a lowprobability stimulus did not necessarily bias the birds to select the comparison stimulus associated with the higher probability of reinforcement. Accuracy on high- and lowprobability trials declined equally as the DI was increased in Experiment 2. Even if the birds had a bias towards the high-probability stimulus and guessed more often as the DI was increased, accuracy should have decreased less rapidly on high-probability trials than on low-probability trials. The parallel effect of DI on these trials suggests that there was no bias.

In Experiments 1, 3, and 4, with short DI durations, an effect of ITI duration was observed for low-probabilityof-reinforcement trials but not for high-probability-ofreinforcement trials. Only in Experiments 3 and 4 at a DI of $15 \mathrm{sec}$ was an effect of ITI duration obtained on high-probability-of-reinforcement trials. In agreement with the findings of Spetch (1984) and Wilkie (1984), Experiment 4 demonstrated that unsignaled ITI reinforcers disrupt DMTS accuracy. This disruption only occurred when the DI was long $(15 \mathrm{sec})$ and when the ITI was short $(5 \mathrm{sec})$.

According to scalar expectancy theory, the ratio of expectancy during trial stimuli to background expectancy is equivalent to the ratio of cycle time (average time between reinforcers) to trial duration (time from warning signal to the reinforcer). Numerical values of the ratio of cycle time to trial duration for various conditions in the present studies are presented in Table 4 . These ratios were calculated assuming errorless performance and a trial duration equal to $7 \mathrm{sec}+$ DI duration (1-sec warning light, 5-sec sample, 1-sec comparison). If trial spacing effects were dependent on a comparison of the average level of expectancy during trial stimuli with the background level of expectancy, then the value of the expectancy ratios for the DO group on both high- and lowprobability trials would be the same and numerically equivalent to those given for the NDO group in Table 4. As a result, similar trial spacing effects should be obtained in both DO and NDO groups, and within the DO group, on both high- and low-probability trials. The differential sensitivity of high- and low-probability trials to ITI duration is not consistent with this theoretical approach.

An alternative approach might be to assume that the level of expectancy during high- and low-probability trials 
Table 4

The Ratio of Cycle Time to Trial Duration for Various Conditions

\begin{tabular}{ccccccc}
\hline & & \multicolumn{3}{c}{ Cycle Time/Trial Duration } \\
\cline { 3 - 6 } Condition & Probability of & \multicolumn{2}{c}{ DI 5 sec } & & \multicolumn{2}{c}{ DI 15 sec } \\
\cline { 3 - 6 } \cline { 3 - 6 } & Reinforcement & ITI 1 sec & ITI 25 sec & & ITI 1 sec & ITI 25 sec \\
\hline DO & 1.0 (high) & 1.81 & 5.14 & & 1.74 & 3.56 \\
& 0.2 (low) & 0.36 & 1.03 & & 0.35 & 0.71 \\
NDO & 0.6 & 1.09 & 3.09 & & 1.05 & 2.14 \\
\hline
\end{tabular}

is compared independently with the background level of expectancy. In this case, as shown in Table 4, the values of the ratios would differ for high- and low-probability trial types, and these would differ from the ratios for the NDO group. Note that, in all conditions, as the ITI duration is shortened, the value of the ratio declines. However, for the DO group on high-probability-of-reinforcement trials, the value of the ratio is still quite high at the short ITI relative to the ratios in the other conditions. One might attempt to argue that on high-probability-of-reinforcement trials, the value of the ratio is still great enough to support a high level of accuracy even at a short ITI. Unfortunately, the ratios at a 1-sec ITI are fairly similar for DIs of 5 and $15 \mathrm{sec}$. The occurrence of a trial spacing effect on high-probability trials at a long but not a short DI does not appear to be well represented by this theoretical approach. Furthermore, a comparison of the ratios across conditions indicates that performance should be better for the NDO group than for the DO group on low-probability-of-reinforcement trials. The present data revealed that accuracy was higher for the DO group on low-probability trials than for the NDO group (see Experiment 2). A similar result was reported by DeLong and Wasserman (1981). These results, in conjunction with the finding that the disruptive effect of ITI reinforcers is dependent on their temporal proximity to trial events (see Experiment 4; Spetch, 1984), are difficult to encompass within a scalar expectancy theory framework.

The initial findings of Roberts and Kraemer (1982, 1984) encouraged the application of scalar expectancy theory to DMTS, since accuracy increased as a linear function of $\log$ ITL/delay, and constant ratios of ITI/delay produced by different values of ITI and delay yielded the same levels of accuracy. Further findings that the average ITI of a session controls performance (Roberts \& Kraemer, 1982) and that ITI reinforcers lower DMTS accuracy (Spetch, 1984; Wilkie, 1984) also supported an extension of scalar expectancy theory to DMTS. Unfortunately, the studies presented here indicate that a finegrain analysis of the effects of ITI, delay, and ITI reinforcers on DMTS when trials involve differential outcomes does not consistently support scalar expectancy theory. However, it is possible that some of the present data which were not consistent with scalar expectancy theory may have been subject to ceiling effects. One might argue that performance on high-probability trials at the short DI was so accurate that an increase in accuracy at long ITI durations could not be revealed. Although it is difficult to convincingly rule out the possibility that ceiling effects were present in the data, the assessment of their significance depends upon the frame of reference adopted in examining the data. If one begins with performance at the short ITI and anticipates an increment in accuracy as ITI is lengthened, then ceiling effects appear important. However, if one begins with performance at the long ITI and anticipates a decrement in accuracy as the ITI is shortened, then ceiling effects do not appear important. In Experiment 1, at the long ITI duration, performance on high-probability-of-reinforcement trials was very similar at both the short and long DI. Consequently, it might be expected that shortening the ITI duration would produce a decrement in accuracy on these trials with both the short and the long DI. The decrement was observed only when the DI was long. From this perspective, the failure to obtain the decrement when the DI was short does not appear to be due to a ceiling effect.

In summary, we believe that the present data present problems for a scalar expectancy theory approach to DMTS performance. In part, these problems may reflect the differences between acquisition measures and steady state measures. Scalar expectancy theory accounts for acquisition data adequately, but appears incomplete with respect to autopecking maintenance data (Balsam \& Schwartz, 1981). The present DMTS data reflect steady state performance. Perhaps scalar expectancy theory bolstered by some yet unknown theoretical mechanisms might yet account for these findings. In lieu of this possibility, however, we may have to seek yet another account of trial spacing effects in DMTS.

\section{REFERENCES}

Balsam, P. D., \& SchwarTz, A. L. (1981). Rapid contextual conditioning in autoshaping. Journal of Experimental Psychology: Animal Behavior Processes, 7, 382-393.

Brodigan, D. L., \& Peterson, G. B. (1976). Two-choice conditional discrimination performance in pigeons as a function of reward expectancy, prechoice delay, and domesticity. Animal Learning \& Behavior, 4, 121-124.

Cook, R. G. (1980). Retroactive interference in pigeon short-term memory by reduction in ambient illumination. Joumal of Experimental Psychology: Animal Behavior Processes, 6, 326-338.

DeLong, R. E., Wasserman, E. A. (1981). Effects of differential reinforcement expectancies on successive matching-to-sample performance in pigeons. Journal of Experimental Psychology: Animal Behavior Processes, 7, 394-412.

DURLACH, P. J. (1983). The effect of signaling intertrial USs in autoshaping. Joumal of Experimental Psychology: Animal Behavior Processes, 9, 374-389. 
Edwards, C. A., Jagielo, J. A., Zentall, T. R., \& Hogan, D. E. (1982). Acquired equivalence and distinctiveness in matching to sample by pigeons: Mediation by reinforcer-specific expectancies. Journal of Experimental Psychology; Animal Behavior Processes, 8, 244-259.

GibBoN, J. (1977). Scalar expectancy theory and Weber's law in animal timing. Psychological Review, 84, 279-325.

Gibbon, J., Balsam, P. (1981). Spreading association in time. In C. M. Locurto, H. S. Terrace, \& J. Gibbon (Eds.), Autoshaping and conditioning theory (pp. 219-253). New York: Academic Press.

Grant, D. S. (1975). Proactive interference in pigeon short-term memory. Joumal of Experimental Psychology: Animal Behavior Processes, 1, 207-220.

Grant, D. S., RoberTs, W. A. (1976). Sources of retroactive inhibition in pigeon short-term memory. Joumal of Experimental Psychology: Animal Behavior Processes, 2, 1-16.

Hogan, D. E., Edwards, C. A., Zentall, T. R. (1981). Delayed matching in the pigeon: Interference produced by the prior delayed matching trial. Animal Learning \& Behavior, 9, 395-400.

Honig, W. K., Matheson, W. R., \& Dodd, P. W. D. (1984). Outcome expectancies as mediators for discriminative responding. Canadian Journal of Psychology, 38, 196-217.

Honig, W. K., \& Thompson, R. K. R. (1982). Retrospective and prospective processing in animal working memory. In G. H. Bower (Ed.), The psychology of leaming and motivation: Advances in research and theory (Vol. 16, pp. 239-283). New York: Academic Press.

Jenkins, H. M., Barnes, R. S., \& Barrera, F. J. (1981). Why autoshaping depends on trial spacing. In C. M. Locurto, H. S. Terrace, \& J. Gibbon (Eds.), Autoshaping and conditioning theory (pp. 255-284). New York: Academic Press.

MakJ, W. S., MoE, J. C., \& Bierley, C. M. (1977). Short-term memory for stimuli, responses and reinforcers. Journal of Experimental Psychology: Animal Behavior Processes, 3, 156-177.

Peterson, G. B. (1984). How expectancies guide behavior. In H. L. Roitblat, T. G. Bever, \& H. S. Terrace (Eds.), Animal cognition (pp. 135-148). Hillsdale, NJ: Erlbaum.

Peterson, G. B., Trapold, M. A. (1980). Effects of altering outcome expectancies on pigeons' delayed conditional discrimination performances. Learning \& Motivation, 11, 267-288.

Peterson, G. B., Wheeler, R. L., A Armstrong, G. D. (1978). Expectancies as mediators in the differential-reward conditional discrimination performance of pigeons. Animal Learning \& Behavior, 6 , 279-285.
Peterson, G. B., Wheeler, R. L., \& Trapold, M. A. (1980). Enhancement of pigeons' conditional discrimination performance by expectancies of reinforcement and non-reinforcement. Animal Leaming \& Behavior, 8, 22-30.

ROBERTS, W. A. (1980). Distribution of trials and intertrial retention in delayed matching-to-sample with pigeons. Journal of Experimental Psychology: Animal Behavior Processes, 6, 217-237.

RoberTs, W. A., \& GRANT, D. S. (1978). An analysis of light-induced retroactive inhibition in pigeon short-term memory. Journal of $E x$ perimental Psychology: Animal Behavior Processes, 4, 219-236.

Roberts, W. A., \& KraEmer, P. J. (1982). Some observations of the effects of intertrial interval and delay on delayed matching-to-sample in pigeons. Journal of Experimental Psychology: Animal Behavior Processes. 8, 342-353.

Roberts, W. A., Kraemer, P. J. (1984). Temporal variables in delayed matching to sample. In J. Gibbon \& L. Allan (Eds.), Timing and time perception (pp. 335-345). New York: Annals of the New York Academy of Sciences.

RoItBLAT, H. L., \& SCOPATZ, R. A. (1983). Sequential effects in pigeon delayed matching-to-sample performance. Journal of Experimental Psychology: Animal Behavior Processes, 9, 202-221.

SANTI, A. (1984). The trial spacing effect in delayed matching-to-sample by pigeons is dependent upon the illumination condition during the intertrial interval. Canadian Journal of Psychology, 38, 154-165.

SPETCH, M. L. (1984, June). Effect of inter-trial food presentation on pigeons' short-term memory. Paper presented at the meeting of the Canadian Psychological Association, Ottawa.

Thompson, R. K. R., VAN Hemel, P. E., Winston K. M., \& PapPas, N. (1983). Modality specific interference with overt mediation by pigeons in a delayed discrimination task. Learning \& Motivation, 14, 271-303.

Tranberg, D. K., \& Rilung, M. (1980). Delayed-interval illumination changes interfere with pigeon short-term memory. Joumal of the Experimental Analysis of Behavior, 33, 39-49.

WILKIE, D. M. (1984). Pigeons' spatial memory: IV. Effects of intertrial manipulations on delayed matching of key location. Canadian Journal of Psychology, 38, 178-195.

(Manuscript received January 2, 1985; revision accepted for publication July $22,1985$. 